\title{
NEW TECHNOLOGIES AND THEIR INTERCONNECTION IN THE CREATION AND PROCESSING OF 3D MODELS AND SCENES
}

\author{
M. Janovský ${ }^{1}$ \\ ${ }^{1}$ Czech Technical University in Prague, Faculty of Civil Engineering, Department of Geomatics - michal.janovsky@ fsv.cvut.cz
}

\author{
Commission V, WG V/7
}

KEY WORDS: 3D model, 3D scene, landscape, visualization, new technologies, game engine.

\begin{abstract}
:
This article focuses on the content and processing of the prepared dissertation thesis, which deals with the possibilities of using new technologies and software tools such as procedural generation of 3D models, game engines, rendering programs, virtual and augmented reality, eye tracking and BIM in cartographic processing and 3D landscape visualization. The dissertation focuses on the interconnection of these technologies, on new methods of processing and on the combination of the use of modern software equipment with already existing methods of processing into one complete process. For these purposes, the possibilities of using, among other things, software development kits (SDK), plugins and extensions of existing programs will be explored. Great emphasis will be placed on the Unity game engine and the ability to use its individual parts (rendering engine, physics engine, scripting, simulation, and AI) to create new workflows and new types of processing / outputs. The main goal of this dissertation is to find and document the possibilities of interconnection of individual technologies, especially those that are not commonly used or can be considered new (no scientific articles were found that would use these methods of interconnection). The result of the dissertation will be new working procedures and more realistic visualizations of 3D models and scenes corresponding to the technologies available today. An equally important part of the dissertation will be the creation of sample projects for individual programs, test data, work procedures and general teaching materials to simplify the use of these technologies in teaching and in departmental projects.
\end{abstract}

\section{INTRODUCTION}

Due to the rapid development of technologies, especially in the field of working with $3 \mathrm{D}$ data, it is increasingly difficult to orientate in these technologies and effectively integrate them into existing workflows. Another problem is then trying to effectively combine these new technologies.

For these reasons, this work focuses on mapping new technologies and software tools and their possibilities of interconnection and interconnection with existing processing methods, both theoretically in the form of technology interconnection diagrams, determination of possible inputs and outputs, research of necessary hardware and software, and practically in the form of tutorials, lists of required plugins, extensions and file formats needed to link these technologies.

The aim of this work is to raise awareness of new technologies that are not yet widespread in the field of cartography, facilitate the use of these new technologies and software and facilitate their integration and integration into existing workflows, departmental projects, student theses and teaching in general.

Due to the scope and concept of the work, it is necessary to draw information from many sources that focus on the use of these new technologies in cartography and in general when working with $3 \mathrm{D}$ data. One of the basic resources are scientific articles and conference papers, but in addition to them, other resources will be used, which are focused on specific user experiences and problem solving, such as discussion forums, technical support for individual software, documentation, etc.,

Selected research technologies include procedural model generation, game engines, rendering software, virtual and augmented reality, and eye tracking. This list may be supplemented by other technologies during processing.

\section{TECHNOLOGIES}

The next chapter deals with individual technologies and their use in cartography with a focus on connecting / combining technologies to create more complex outputs.
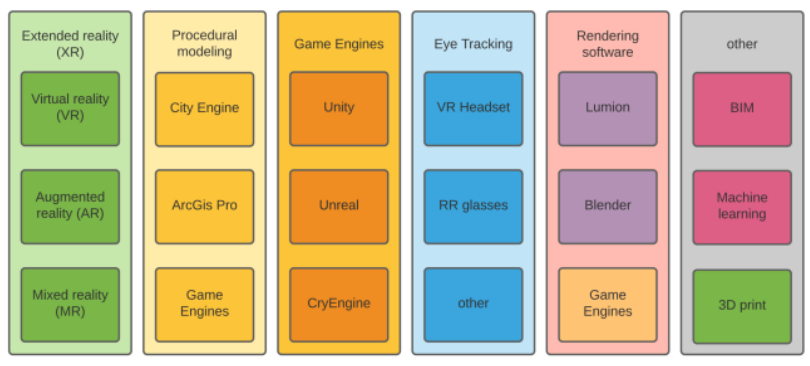

Figure 1. Overview of technologies and their software solutions

\subsection{Procedural 3D modeling}

The first of the examined technologies is the procedural modeling of $3 \mathrm{D}$ models. It is an algorithmic method of 3D modeling according to the so-called "rule file" (Müller \& Wonka, 2006) and (Watson \& Muller, 2008).

This technology is most often used in the visualization of historical data, based on map data (Janovský, Janata, Cajthaml, 2020), (Arnold, Lafreniere, 2018), plans, photographs, and other documents (Kitsakis, Tsiliakou, 2017). It is also used as a method 
for generating models in the vicinity of areas of interest, which no longer need to be processed in such high detail and serves as a complement to the visualization of the environment.

Most scientific papers with procedural generation have one thing in common, that they do not only use outputs from procedural generation, but also use outputs from other technologies such as GIS (Janovský, Janata, Cajthaml, 2020), (Arnold, Lafreniere, 2018), and that procedural modeling results are often used for further processing by other technologies such as game engines (Mueller, 2018 and 2020).

An interesting phenomenon are ESRI's products, namely ArcGIS Pro (GIS software), City Engine (software primarily focused on procedural modeling) and ArcGIS Online (online publishing and visualization platform), where individual technologies are connected directly within ESRI products. Furthermore, conference presentations and papers dealing with the interconnection of CityEngine with game engines (Unity and Unreal) regularly appear at official ESRI conferences (Esri Developer Summit) focusing on improving scene visualizations and VR utilization (Mueller, 2018 and 2020).

\subsection{Game Engines}

Another of the researched technologies are game engines and their use in the processing of 3D models and GIS data with a focus on visualization of results (Mat, 2014). The game engine is a software framework primarily designed for video game development and generally includes relevant libraries and supporting programs, which may include a rendering engine for 2D or 3D graphics, physics engine, collision detection, sound, scripting, animation, artificial intelligence, networking, streaming, memory management, threads management, localization support, scene graphs, and video support for cinematography (Unity, Unreal Engine, 2022). Due to the large number of processing options, game engines are increasingly used for data management (Ma, 2021).

Two of the most used game engines have a function for importing GIS data (Unreal uses the so-called "Datasmith Importer", Unity requires a paid $\mathrm{PiXYZ}$ plugin). In cartography, one of the most frequently used functions of game engines is the creation of VR applications (Mueller, 2020).

\subsection{Virtual reality}

Virtual reality that can be created in game engines is generally a virtual environment with which we can interact using a VR set, ie VR glasses that allow us to see and experience virtual reality and controllers used to interact and move in the virtual world.

VR is used primarily as a form of visualization of 3D models and scenes (Lütjens, Kersten, Dorschel, 2019), but it can also be used for the purpose of optimizing work activities where knowledge from virtual reality is evaluated and the results are then reflected in the real world (Muhammad, Yitmen, Alızadehsaleh1, 2020). Quite often a 3D model or scene is first created using laser scanning or other technology capturing real world data, then imported into a game engine where a VR application is created (Büyüksalih, Kan, Özkan, 2020).

\subsection{Eye-Tracking}

One of the new technologies is also Eye-Tracking, which takes several forms: static and mobile (Pentus, Ploom, Mehine, 2020), and the latest eye-tracking in VR (Clay, P. König, S. U. König, 2019). The principle of eye-tracking is to monitor and record eye movement, this data is then stored and statistically processed. During the processing, the length and frequency of fixation of the view of the objects of interest are evaluated, from which conclusions are drawn related to the observed phenomenon (Niehorster, Santini, Hessels, 2020).

In cartography, eye-tracking is used mainly to evaluate cartographic works in terms of readability and understanding, which is mainly addressed by RNDr. Stanislav Popelka, Ph.D. (Popelka, 2012, 2013, 2018).

\subsection{Rendering software}

Another large group of technologies is rendering software, which focus on creating a so-called "render". Render is a photorealistic representation of images, scenes and models by working with materials (textures), lighting, shading, filters and other attributes that are applied to the original data by a computer process (Shum, Kang, 2000) (Botsch, Kobbelt, 2003). Game engines that contain their own rendering engine can also be freely included in the category of rendering software (Baek, Ji, Jin, Yun, 2019). Many different rendering software are used in practice. Freely available Blender or paid Lumion (Ramdhaniati, 2021) are often used in cartography.

\subsection{Other technologies}

Other technologies such as 3D printing for the creation of physical models (Oswald, 2019), BIM and machine learning will be gradually incorporated into this work during its course.

\section{METHOD}

The whole work consists of three parts:

The first part deals with the theory of individual technologies, the possibilities of their use in the visualization of 3D models and scenes, the possibilities of interconnection with other technologies, input and output formats, hardware and licensing requirements for use in teaching, and comparison of software solutions.

The second part deals with theoretical and practical possibilities of interconnecting technologies with a focus on variables that may affect this process (file formats, data size, data type, software used, planned further processing, selected plugins and extensions, etc.)

The third part consists of the creation of teaching materials to connect and work with individual technologies and software. For the purposes of the dissertation, however, it will rather serve only as an example of possible results and work processes arising from the interconnection of technologies and solving the problems that have arisen. 


\subsection{Solution procedure}

To facilitate the solution of the use and interconnection of technologies, individual steps have been set according to which the examination of these technologies will proceed. The following points will be tested for each technology and its software solution:

1) analyze possible uses for cartographic visualization

2) find the best way to import different types of data

3) analyze the suitability for processing large amounts of data (both in size and quantity)

4) process test data

5) compare processing and results with other programs with the same functionality

6) find other technologies that could follow the processing to obtain new or improved results

7) document the whole process

8) create teaching materials / test projects

9) find / design / process new workflows / outputs

10) graphical display of points 1-9 in the form of flowcharts

\subsection{Survey of individual technologies}

The first part of the work focuses on the research of various technologies that can be used in the processing of 3D models and scenes. These technologies and software examine the types and formats of inputs and outputs, the theoretical possibilities of interconnection with other technologies, the necessary hardware and software for the use of individual technologies and, if possible, the use of multiple software or hardware solutions by comparing them.

During the literature search and preparatory work in the research of individual technologies, various research goals for individual technologies were found and set.

- Focus on technical issues associated with interconnecting of technologies, such as the interconnection of CityEngine (procedural modeling) and game engines, where file formats play a major role, such as the appropriateness of using specific technologies and software for big data processing.

- Explore the possibilities of using plugins, extensions and software development kits (SDK) of existing programs, selecting the appropriate engine, comparing functionalities with other technologies, such as comparing model rendering and creating VR applications in game engines and rendering software such as Blender or Lumion, which also have these features.

- Great emphasis will be placed on the Unity game engine and the ability to use its individual parts (rendering engine, physics engine, scripting, simulation, and AI) to create new workflows and new types of processing / outputs

- In virtual reality, explore the choice of software, optimization of data, models and scenes that will be used as a basis for virtual reality and the possibilities of linking virtual reality with eye-tracking, which will allow the acquisition of visual data in VR.
- Compare the functionality and outputs of rendering software and game engines and determine when and how to choose between these technologies / software with respect to input data variables.

- Focus on the applied use of eye-tracking for better creation of cartographic 3D visualizations, specifically to explore the possibilities of connecting eye-tracking with the process of creating 3D models and scenes. Using eye-tracking data processing results as input data for creating and editing 3D models and scenes.

- Use mobile eye tracking to improve the quality of 3D printed models and generally examine the adjustments to the model and input data required for physical 3D printing.

\subsection{Technology interconnection}

Diagrams showing the possibilities of processing, individual outputs, possibilities of visualization of outputs and possibilities of using outputs by other technologies (programs) are first created for individual technologies. These diagrams are created iteratively, as with each new technology the diagrams of other technologies are extended by the possibility of interconnection or new processing possibilities (optimization of processing for further use by another technology, etc.).

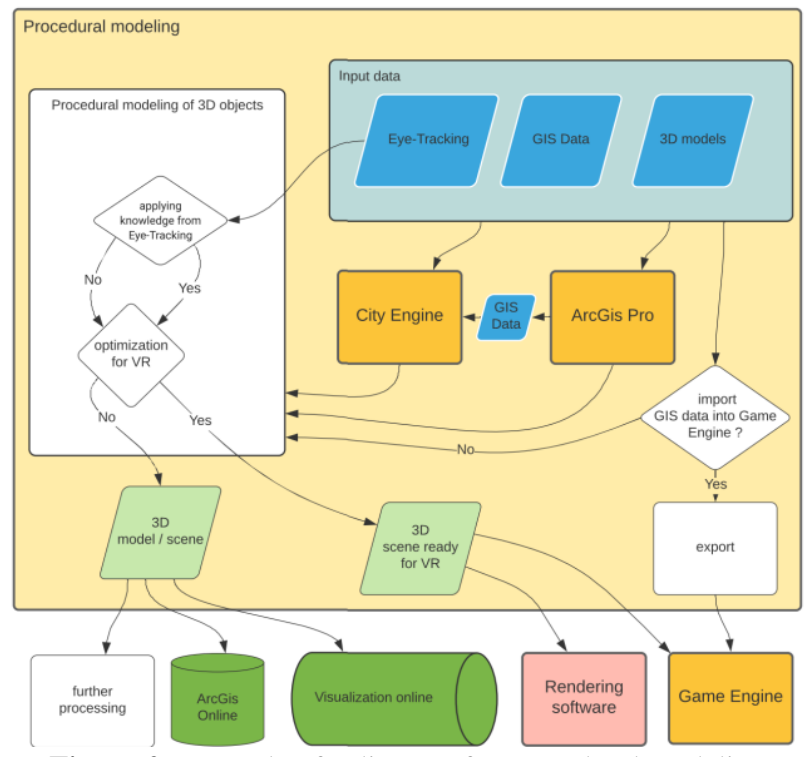

Figure 2. Example of a diagram for procedural modeling

An example of a flowchart is shown in Figure 2, which shows that procedural modeling involves creating a 3D model that we can optimize for VR (since we look at the model in VR from a different perspective and some parts of the model are visible) and the results can be exported in formats suitable for import into game engines. Furthermore, we can increase the quality (detail) of models in areas of interest and reduce the quality in other areas, while data on these sites can be obtained from statistical data obtained from the processing of data from Eye-Tracking. All these steps are optional, and it only makes sense to perform them if we want to link procedural modeling with these other technologies, and according to our choice and needs, the whole next workflow will change. 
After compiling the diagrams of individual technologies, the second step occurs - Interconnection of technologies. This step specifically examines how to connect the various technologies, from simple data export and import to using user experience to select appropriate plugins, extensions, and the most appropriate file formats for data import. When using several software solutions, it is further investigated which software to use and why.

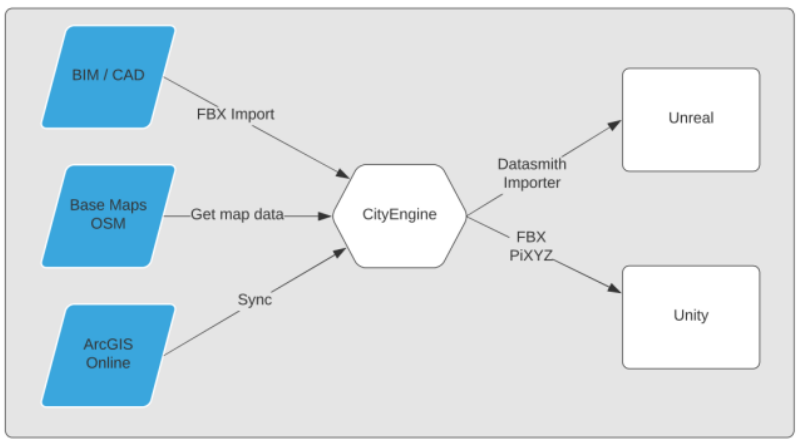

Figure 3. Scheme of importing GIS data into game engines

As with the survey of individual technologies, flowcharts are created here as well. These diagrams show the possibilities of interconnecting technologies. Due to the influence of input data on the possibilities of processing and interconnection of technologies, several versions of these diagrams will be created, which will represent different input data (size, type, format, etc.).

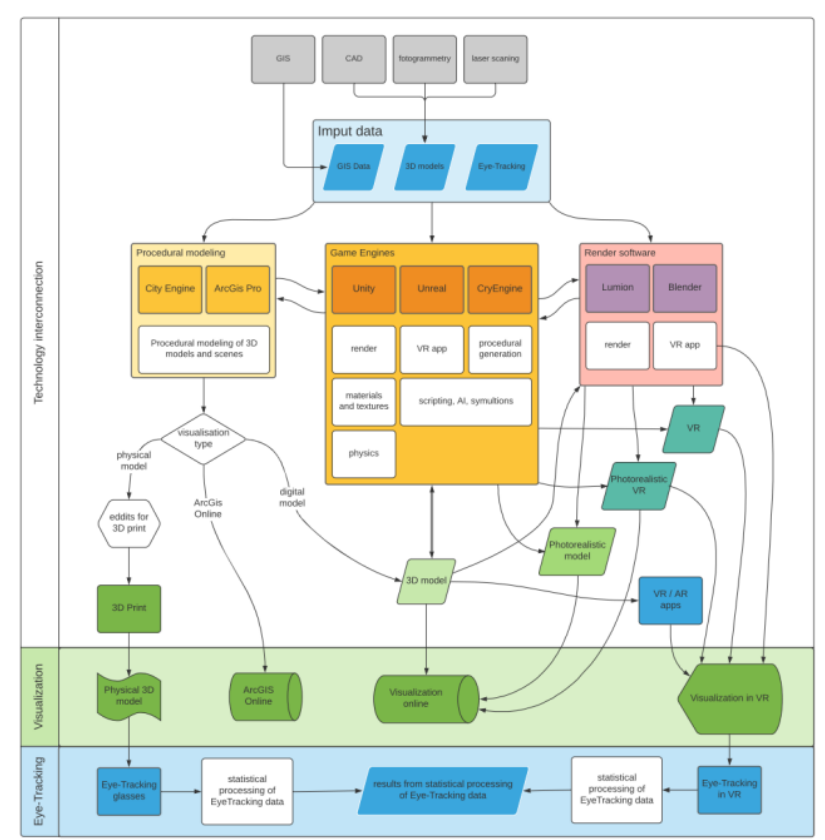

Figure 4. Example of a diagram for technology interconnection

Figure 4 shows a diagram with theoretical possibilities of interconnection of individual technologies. What is missing here, however, are the specific steps needed to link them, which are often much more complicated than they might seem and vary depending on many factors.

As an example, there is a figure 3 , which shows the procedure of importing GIS data into the two most used game engines (Unity and Unreal). In this case, the CityEngine program is used as an intermediary for import, as there are generally problems with importing GIS data (most often in FBX format), even in cases where this option is directly built into the game engine (Unreal). The second of the engines (Unity) supports the import of GIS data only using plugins such as paid PiXYZ.

To prevent these problems, CityEngine is used, in which it is possible to create a scene with data that is imported much easier and without major problems. In this example, it is clear how much depends on specific other software solutions, data format, and variables that significantly affect the interconnection of individual technologies.

\subsection{Processing test data}

During the whole work, it is necessary to practically test individual work procedures and ideas about working with individual technologies and their interconnection. Although the practical part of this work is not intended as a case study for the processing of specific data, but as a general study whose results should be applicable in general, one of the results should be teaching materials demonstrating findings and specific procedures on specific data.

These teaching materials are used to introduce the various technologies and software associated with them, and their main task is to facilitate the use of these technologies in professional projects and in teaching.

Teaching materials will take the form of training projects and data, video tutorials, links to the necessary software, extensions, plugins, etc.

Content of teaching materials:

- Pre-prepared test data and projects

- Documentation of the possibilities of using individual technologies / software

- Documentation of basic functions usable for data processing

- $\quad$ Specific procedures for data import / export

- Solving common problems

- Specific procedures for interconnection of individual technologies

- Documentation of possibilities of further processing and visualization of results

\section{RESULTS}

The main goal of this dissertation is to find and document the possibilities of interconnection of individual technologies, especially those that are not commonly used or can be considered new (no scientific articles were found that would use these methods of interconnection).

The result of the dissertation will be new working procedures and more realistic visualizations of $3 \mathrm{D}$ models and scenes corresponding to the technologies available today. An equally important part of the dissertation will be the creation of sample projects for individual programs, test data, work procedures and general teaching materials to simplify the use of these technologies in teaching and in departmental projects. 
The expected results of this work are described in several points below

- Overview of available technologies working with 3D data

- Comparison of software within individual technologies

- Flowcharts of 3D data processing / visualization

- Practical solution for connecting individual technologies

- Flowcharts of interconnection of individual technologies

- Teaching materials to connect and use individual technologies

\section{CONCLUSION}

This study serves to present the planned content of the prepared dissertation. The study includes a literature search on individual technologies and their use and interconnection when working with 3D data. Most of the published works concerning the use of new technologies in cartographic processing and visualization of 3D data deal with a specific problem and use specific technologies to solve it. The combination of technologies is much less common, especially in the cartographic processing of $3 \mathrm{D}$ visualizations.

This planned dissertation deals with the use of many technologies at once and instead of a specific problem addresses the general possibilities of interconnection and use of technologies and examines the possible results resulting from the combination of these technologies.

The dissertation will map the processes of working with 3D visualizations using new technologies, which will be presented, among other things, in the form of flowcharts. These processes will be analyzed in detail with a focus on the impact of input data, problematic places of processing will be found and described, and methodological solutions to specific processing problems will be developed.

The main benefit of this work will be a broader methodological view of the whole issue of using and connecting new technologies in the creation and processing of 3D models and scenes in cartographic processing and 3D landscape visualization. This broader view should make it easier and better to use new technologies and should provide a "guide" to combining technologies and creating better landscape visualizations.

\section{REFERENCES}

Baek, KH; Ji, Y; Jin, HW; Yun, TS; "Game Engine PBR for Background CGI Production of Live-action Contents," 2019 IEEE Conference on Graphics and Media (GAME), 2019, pp. 1821, https://doi.org/10.1109/GAME47560.2019.8980984

Botsch, M.; Kobbelt, L.; "High-quality point-based rendering on modern GPUs," 11th Pacific Conference onComputer Graphics and Applications, 2003. Proceedings., 2003, pp. 335-343, https://doi.org/10.1109/PCCGA.2003.1238275

Brychtová, A., Popelka, S., \& Vozenilek, V. (2012, September). The analysis of eye movement as a tool for evaluation of maps and graphical outputs from GIS. In Proceedings of the 19th International Conference on Geography and Geoinformatics:
Challenge for Practise and Education. Brno, Czech Republic (pp. 154-162).

https://katedry.ped.muni.cz/geografie/wp-

content/uploads/sites/8/2013/10/sbornik-tisk.pdf

Büyüksalih, G., Kan, T., Özkan, G.E. et al. Preserving the Knowledge of the Past Through Virtual Visits: From 3D Laser Scanning to Virtual Reality Visualisation at the Istanbul Çatalca İnceğiz Caves. $P F G$ 88, 133-146 (2020).

https://doi.org/10.1007/s41064-020-00091-3

Che Mat, PhD, Ruzinoor \& shariff, rashid \& Zulkifli, Abdul Nasir \& Rahim, Mohd \& Hafiz, Mohd. (2014). Using game engine for 3D terrain visualisation of GIS data: A review. IOP Conference Series: Earth and Environmental Science. 20. 012037. 10.1088/1755-1315/20/1/012037.

https://iopscience.iop.org/article/10.1088/17551315/20/1/012037

Clay, V., P. König, and S. U. König. "Eye Tracking in Virtual Reality". Journal of Eye Movement Research, vol. 12, no. 1, Apr. 2019,https://doi.org/10.16910/jemr.12.1.3

Docs.unity.com. n.d. Unity Documentation. [online] [Accessed 28 November 2021]. Available at: https://docs.unity.com

Docs.unrealengine.com. n.d. Unreal Engine 4 Documentation. [online] [Accessed 28 November 2021]. Available at: https://docs.unrealengine.com/4.27/en-US/

Janovský, M.; Janata, T.; Cajthaml, J.; Visualization of the Vltava River valley: illustration of work procedures on data from the Kamyk reservoir surroundings; In: 20th International Multidisciplinary Scientific GeoConference SGEM 2020. Sofia: STEF92 Technology Ltd., 2020. p. 469-476. ISSN 1314-2704. ISBN 978-619-7603-07-1.

http://dx.doi.org/10.5593/sgem2020/2.2/s11.056

John David McEwen Arnold, Don Lafreniere, (2018) "Creating a longitudinal, data-driven 3D model of change over time in a postindustrial landscape using GIS and CityEngine", Journal of Cultural Heritage Management and Sustainable Development, Vol. 8 Issue: 4, pp.434-447,

https://doi.org/10.1108/JCHMSD-08-2017-0055

Kitsakis, D., Tsiliakou, E., Labropoulos, T., and Dimopoulou, E.: Procedural 3D modelling for traditional settlements. The case study of central Zagori, Int. Arch. Photogramm. Remote Sens. Spatial Inf. Sci., XLII-2/W3, 369-376, 2017 https://doi.org/10.5194/isprs-archives-XLII-2-W3-369-2017

Lütjens M, Kersten TP, Dorschel B, Tschirschwitz F. Virtual Reality in Cartography: Immersive 3D Visualization of the Arctic Clyde Inlet (Canada) Using Digital Elevation Models and Bathymetric Data. Multimodal Technologies and Interaction. 2019; 3(1):9. https://doi.org/10.3390/mti3010009

Ma Y-P. Extending 3D-GIS District Models and BIM-Based Building Models into Computer Gaming Environment for Better Workflow of Cultural Heritage Conservation. Applied Sciences. 2021; 11(5):2101. https://doi.org/10.3390/app11052101

Muhammad, A. A. , Yitmen, İ. , Alızadehsalehı, S. , Celık, T. "Adoption of Virtual Reality (VR) for Site Layout Optimization of Construction Projects" . Teknik Dergi 31 (2020 ): 9833-9850 https://doi.org/10.18400/tekderg.423448 
Müller, Pascal \& Wonka, Peter \& Haegler, Simon \& Ulmer, Andreas \& Van Gool, Luc. (2006). Procedural Modeling of Buildings. ACM Trans. Graph.. 25. 614-623. https://doi.org/10.1145/1141911.1141931

Niehorster, D.C., Santini, T., Hessels, R.S. et al. The impact of slippage on the data quality of head-worn eye trackers. Behav Res $52,1140-1160$ (2020). https://doi.org/10.3758/s13428-019-01307-0

Oswald, Claire, et al. "Applications of 3D Printing in Physical Geography Education and Urban Visualization." Cartographica: The International Journal for Geographic Information and Geovisualization, vol. 54 no. 4, 2019, p. 278-287. Project MUSE muse.jhu.edu/article/743804

Pascal Mueller (March 7-10, 2018). High-End 3D Visualization with CityEngine, Unity and Unreal [Conference presentation]. 2017 Esri Developer Summit, Palm Spring, CA, United States. https://proceedings.esri.com/library/userconf/devsummit18/inde x.html

Pascal Mueller, Stefan Arisona (March 10-13, 2020). Building VR/AR Experiences with CityEngine, Unity, and Unreal [Conference presentation]. 2020 Esri Developer Summit, Palm Spring, CA, United States.

https://proceedings.esri.com/library/userconf/devsummit20/inde $\mathrm{x} . \mathrm{html}$

Pentus, K., Ploom, K., Mehine, T., Koiv, M., Tempel, A. and Kuusik, A. (2020), "Mobile and stationary eye tracking comparison - package design and in-store results", Journal of Consumer Marketing, Vol. 37 No. 3, pp. 259-269. https://doi.org/10.1108/JCM-04-2019-3190

Popelka, S. . Eye-tracking evaluation of 3D thematic maps. In Proceedings of the 3rd Workshop on Eye Tracking and Visualization (ETVIS '18). Association for Computing Machinery, 2018, New York, NY, USA, Article 5, 1-5. https://doi.org/10.1145/3205929.3205932

Popelka S, Brychtova A. Eye-tracking study on different perception of 2D and 3D terrain visualisation. The Cartographic Journal. 2013 Aug 1;50(3):240-6. https://doi.org/10.1179/1743277413Y.0000000058

Ramdhaniati, M \& Mulyanti, Budi. (2021). Lumion animation for digital learning: Is it applicable in both Industrial and educational contexts? IOP Conference Series: Materials Science and Engineering. 1098. 022113.

https://iopscience.iop.org/article/10.1088/1757-

$899 X / 1098 / 2 / 022113$

Shum, HY.; Kang, SB.; "Review of image-based rendering techniques", Proc. SPIE 4067, Visual Communications and Image Processing 2000, (30 May 2000);

https://doi.org/10.1117/12.386541

Watson, Benjamin \& Muller, P. \& Wonka, Peter \& Sexton, Chris \& Veryovka, Oleg \& Fuller, Andy. (2008). Procedural Urban Modeling in Practice. Computer Graphics and Applications, IEEE. 28.18 - 26.

https://doi.org/10.1109/MCG.2008.58 